\title{
Anna Hager* \\ Die Kopten und der Arabische Frühling: Zwischen politischer Emanzipation und Minderheitenstatus
}

https://doi.org/10.1515/asia-2017-0004

\begin{abstract}
The participation of young Copts in the Arab Spring in January 2011 was all the more surprising given the widespread image of a community facing a number of discriminations and being somewhat under siege. This article seeks to highlight the dynamics of a community that had begun to emancipate itself from church tutelage in the context of growing opposition to the regime of Husni Mubārak prior to 2011. In particular, it will take the narrative of national unity as a lens through which to understand how Coptic Egyptians navigated between the status of actors and subjects of various discourses. I consider this narrative a tool which was used by various Christian (the Churches and activists) and nonChristian actors (state, Islamists) to promote their interests. This paper aims to go beyond the issue of sectarian strife and look at how, during this period (2011-2013), Coptic activists came to interact with Islamist actors.
\end{abstract}

Schlüsselwörter: Ägypten, Arabischer Frühling, Kopten, Minderheit, östliches Christentum

\section{Einleitung}

Die Christen im Nahen Osten gelten im Allgemeinen als Befürworter eines stabilen politischen Systems und somit als Gegner des sogenannten Arabischen Frühlings. Insbesondere die christlichen Ägypter wurden lange als eine politisch passive Minderheit betrachtet, die sich aufgrund der Verschlechterung ihrer Lage in den letzten Jahrzehnten, innerhalb der Kirche zurückgezogen hatten, welche als ihr politisches Sprachrohr agierte. Doch unter den „Märtyrern“ der Revolution vom 25. Jänner 2011 befanden sich auch junge Kopten, deren Anwesenheit durch die Verwendung christlicher Symbole - neben islamischen Symbolen - materialisiert wurde.

Ausgehend von diesem vermeintlichen Widerspruch, untersucht dieser Beitrag die Frage, wie die post-revolutionäre Phase (2011-2013) genutzt wurde, um

*Corresponding author: Anna Hager, Institute of Eastern Christian Studies, Radboud University, Nijmegen, Netherlands. E-mail: anna.hager23@gmail.com 
den Status der Kopten (neu) zu definieren. Ich beschränke mich somit auf die Zeit zwischen dem Vorabend der Revolution 2011 und den Sturz von Muhammad Mursī im Juli 2013 und beanspruche, das Spannungsverhältnis zwischen „politischer Emanzipation“ und „Minderheitenstatus“ anhand des Diskuses der nationalen Einheit zu untersuchen. In diesem Zusammenhang stellt die Theorie des kollektiven Gedächtnisses von Maurice Halbwachs („each memory is a viewpoint on the collective memory, that this viewpoint changes as my position changes“1) einen interessanten theoretischen Ansatz dar, um die zahlreichen Spannungsverhältnisse dieser Zeit zu erfassen; die Kopten als Subjekte diverser Diskurse und Akteure, die Polarisierung aber auch die Interaktion zwischen den koptischen Aktivisten und den muslimischen und islamistischen Kräften. Halbwachs Theorie kann dahingehend erweitert werden, um den Diskurs der nationalen Einheit nicht (nur) als Floskel zu betrachten, sondern als Mittel, das sowohl von christlichen (Kirchen, Aktivisten) als auch nicht-christlichen Akteuren (Staat, Islamisten) aus unterschiedlichen Interessen verwendet wurde.

Das theoretische Postulat des Diskurses der nationalen Einheit ermöglicht es, wichtige wissenschaftliche Beiträge zu leisten. Obwohl das Thema der politischen Emanzipation der Kopten im Zuge des Arabischen Frühlings bereits untersucht wurde ${ }^{2}$, enden diese meist mit dem Jahr 2012. In den Jahren 2012 und 2013 ergaben sich jedoch weitere signifikante Dynamiken, die es gilt, hier zu untersuchen, etwa hinsichtlich der politischen Rolle der Kirche. Außerdem tendieren bisherige Untersuchungen dazu, auf die anhaltenden Gewaltausbrüche zwischen Christen und Muslimen zu fokussieren, welche als strukturierendes Element des koptischen Aktivismus betrachtet werden. Dabei spielte die Interaktion mit den islamistischen Kräften - sowohl in Form gegenseitiger Polemik als auch gegenseitiger Unterstützung - eine wichtige Rolle. Bisherige Untersuchungen haben richtigerweise auf die Vielfalt der koptischen Gemeinde hingewiesen, jedoch den Begriff „die Kopten“ selten hinterfragt, obwohl er Teil eines Diskurses ist, wie nachfolgend aufgezeigt wird.

$\mathrm{Zu}$ diesem Zwecke werden offizielle Stellungnahmen, Berichte in allgemeinen, einschlägigen und kirchlichen Medien sowie Interviews, die in Jänner und Februar 2015 in Kairo geführt wurden, heran- und einem historischen und philologischen Ansatz unterzogen. Mit diesem Artikel werden nicht nur inhaltliche Ziele verfolgt, sondern auch, wie weiter oben angeführt, methodologische. Insbesondere sollen der Begriff „die Kopten“ und das Primat der Religion als identitätsstiftender Faktor hinterfragt werden. Die Untersuchung ist in vier Teilen gegliedert. Der erste Teil setzt sich mit dem allgemeinen politischen und

1 Halbwachs 1950 [1950]: 48.

2 Siehe Elsässer 2013; Guirguis 2012; Tadros 2013. 
historischen Rahmen vor und nach der Revolution auseinander. Die anschließenden drei Teile untersuchen das Verhältnis zum Diskurs der nationalen Einheit aus der Perspektive jeweils der Kirchen, des koptischen Aktivismus, sowie des Staates und der islamistischen Kräfte.

\section{Die Rahmenbedingungen vor und nach der Revolution: die Kopten als Minderheit}

In einem ersten Schritt werden die unterschiedlichen Faktoren aufgezeigt, die in der zweiten Hälfte des zwanzigsten Jahrhunderts die Stellung der koptischorthodoxen Kirche als politisches Sprachrohr der christlichen Ägypter etablierten. Dabei ist zu beachten, dass in einem gesamtägyptischen Kontext die politischen Rechte und Partizipationsmöglichkeiten sowohl der Christen als auch der Muslime eingeschränkt waren und dass der Staat die politische Rolle der Kirche zum Nachteil der Christen als Bürger förderte. Dieser erste Teil zeigt auch die wichtigsten Entwicklungen der Jahre 2011-2013 auf.

Der Staatsstreich der Freien Offiziere im Jahre 1952 und insbesondere das Regime von Ğamāl 'Abd al-Nāṣir legten den Grundstein für eine Vormachtstellung der Kirche. Zum einen wurde die Macht der bisherigen politischen und wirtschaftlichen Elite, zu der koptische Notablen gehörten, durch Wirtschaftsreformen - insbesondere die Landreform - gebrochen. Zum anderen wurde auch die Vormachtstellung dieser koptischen Elite innerhalb der christlichen Gemeinde gebrochen, nachdem Ğamāl 'Abd al-Nāṣir das mağlis millì abschaffte $^{3}$. Somit fiel die historisch bedeutende Rolle der koptischen Notabeln weg, die eine wichtige Funktion beim Erhalt des religiösen Erbes und der christlichen Identität spielte ${ }^{4}$ und oft, aber nicht immer, in Konkurrenz zur kirchlichen Hierarchie stand ${ }^{5}$.

Diese neue Machtkonstellation nach der Juli-Revolution in 1952 wurde in Anlehnung an das osmanische System auch als „millet-Partnerschaft“

3 Das mağlis milli wurde 1973 wiedererrichtet und bestand fortan aus Mitgliedern der Mittelschicht (Sedra 2012a).

4 Hier sei auf die tragende Rolle der Familie Awlād al-'Assāl Familie im 13. Jahrhundert hingewiesen, von der ein Teil im Dienste der Ayyubiden tätig war, während ein anderer Teil bedeutende Beiträge im Bereich der Theologie, der Christologie und der Ökumene lieferte (siehe Davis 2008: 252).

5 Febe Armanios weist darauf hin, dass Notabeln und Kirche unter der frühen osmanischen Herrschaft eng miteinander kooperierten, um die religiösen Praxen und die religiöse Identität der Kopten - in allen Gesellschaftsschichten - aufrechtzuerhalten (siehe Armanios 2011). 
bezeichnet, wodurch die Kirche an Bedeutung gewann im Gegenzug für ihre Loyalität gegenüber dem Regime ${ }^{6}$, wobei dies sehr stark von den jeweiligen Persönlichkeiten und politischen Ausrichtungen abhing. Im Falle des Verhältnisses zwischen Patriarch Kyrillos VI. und Ğamāl 'Abd an-Nāṣir einerseits und Šinūda III. und Ḥusnī Mubārak andererseits kann tatsächlich von einer „,milletPartnerschaft“ gesprochen werden, da der Patriarch im Namen der christlichen Ägypter direkt mit dem Staatsoberhaupt verhandelte. Unter Anwar as-Sādāt (1970-1981) verschlechterte sich das Verhältnis. Letzterer trug maßgeblich dazu bei, den politischen Islam als Gegengewicht $\mathrm{zu}$ den sozialistischen und kommunistischen Tendenzen $\mathrm{zu}$ etablieren ${ }^{7}$. Aufgrund der sich häufenden Angriffe auf Kopten und Kirchen in den 1970er Jahren und des zunehmend islamischen Charakters des Staates, verhärtete sich die Position des neuen koptisch-orthodoxen Patriarchen Šinūda III. (1923-1971-2012), der von asSādāt abgesetzt und in ein Kloster verbannt wurde. Gleichzeitig fühlte sich der ägyptische Präsident in seiner Macht und Autorität durch den revolutionären Stil des neuen Patriarchen in Frage gestellt ${ }^{8}$. Die Haltung von Šinūda III. gegenüber Husnī Mubārak änderte sich grundlegend, obwohl der neue Präsident ebenfalls eine ambivalente Haltung gegenüber den islamistischen Kräften unterhielt ${ }^{9}$.

Nicht nur die politischen Entwicklungen hatten eine Auswirkung auf die Lage der Kopten und die Stellung der Kirche, auch die sozialen und demographischen Umwälzungen hatten einen tiefgreifenden Einfluss. Die Landflucht und die Abwanderung in die Städte bewirkten, dass sich die Bevölkerung neumischte, d.h. Kopten siedelten sich in Suez und im Sinai an, wo es früher kaum Christen gab $^{10}$. Somit stellte sich die Frage nach dem Bau neuer Kirchen und nahm immer kontroversere Züge an. Der Beginn der Angriffe auf Kopten und Kirchen wird oft auf das Jahr 1972 zurückgeführt, als eine illegal gebaute Kirche in al-Hānka durch Muslime angriffen wurde und hundert Priester dagegen demonstrierten $^{11}$. Vivian Ibrahim und Sebastian Elsässer weisen jedoch darauf hin, dass es bereits in den 1950er und 1960er Jahren derartige Angriffe gegeben hatte. ${ }^{12}$ Dieses Phänomen nahm in den Jahren vor dem Arabischen Frühling zu

\footnotetext{
6 Siehe McCallum 2012: 109.

7 Roussillon 1994: 105.

8 Siehe Tadros 2013: 67.

9 Unter Mubārak wurden massenhaft Lehrer, Sozialarbeiter, Prediger mit einer „islamischen Gesinnung “ in den Ministerien für Bildung, Soziales und fromme Stiftungen rekrutiert (Roussillon 1994: 106-107). Er spielte außerdem die salafistischen Kräfte gegen die Muslimbruderschaft auf.

10 Reiss 2013: 19.

11 Reiss 1998: 289.

12 Ibrahim 2013, 159; Elsässer 2014: 68.
} 
und ergab sich aus Konflikten um Kirchenbau und -Renovierung, um Land und aus geschlechterbezogenen Konflikte (das Verschwinden oder die Entführung junger koptischer Frauen, interreligiöse Liebesbeziehungen). Dass solche Angriffe und Konflikte euphemistisch als "fitna țā'ifiyya“ (interkonfessionelle Aufruhr, Eng. sectarian strife) bezeichnet werden, wird u.a. vom Publizisten und Herausgeber der christlichen Zeitung Wațani, Yūsif Sīdhum, scharf kritisiert, weil dieser Begriff Opfer und Täter gleichstelle ${ }^{13}$. Die Art und Weise, wie der Staat und die Medien mit solchen Fällen umgingen, verdeutlichte sowohl Sensationsgier als auch Tabuisierung ${ }^{14}$. Einerseits wurden derartige interkonfessionelle Vorfälle medienwirksam präsentiert, da damit mehr Auflagen verkauft wurden $^{15}$. Andererseits wurde auf offizieller, politischer, islamischer und kirchlicher Ebene das Bestehen solcher religiösen Spannungen „zwischen den zwei Elementen der Nation“ negiert ${ }^{16}$. Stattdessen wurde der Diskurs der nationalen Einheit, bei der die Revolution von 1919 als Vorbild gilt, systematisch in den Vordergrund gestellt ${ }^{17}$, und statt strafrechtlicher Verfolgungen, sollten Aussöhnungsversammlungen den sozialen Frieden wiederherstellen.

Am 6. Jänner 2011 - zwei Wochen vor dem Beginn der Proteste - fand ein Anschlag auf eine kirche in Alexandria statt, bei dem über 20 Menschen starben. Gewissermaßen waren die Proteste in Reaktion auf den Anschlag ein wichtiger Wegbereiter für die Revolution. Die Reaktionen auf den Anschlag in Alexandria verdeutlichten abermals einen koptischen Aktivismus, der in den Jahren zuvor begonnen hatte, sich von der politischen Bevormundung durch die Kirche zu emanzipieren. Die Teilnahme von Kopten an der Revolution ist jedoch quantitativ schwer einzuschätzen und war vor allem durch die Einbindung christlicher Symbole ersichtlich, die den Willen bezeugten, sowohl als Ägypter als auch als Kopten teilzunehmen. So fanden mehrere Gedenkfeiern für die Opfer des Anschlages in Alexandria statt, bei der am 9. Februar 2011 zuerst auf Koptisch und anschließend auf Arabisch gebetet wurde ${ }^{18}$, es sangen auch Kirchenchore ${ }^{19}$. Nach der Revolution verdeutlichte sich der Bruch zwischen der Kirchenführung - insbesondere Patriarch Šinūda - und einem Teil der politisch aktiven koptischen Jugend. Vor allem nach dem sogenannten Maspero-Massaker im Oktober 2011 und dem Tod des Patriarchen im März 2012 war die koptisch-orthodoxe Kirche gezwungen, ihre

13 Sidhom 2011.

14 Iskander 2012.

15 Iskander 2012: 32-33.

16 Guirguis 2012: 42-44.

17 Iskander 2012: 32-33.

18 Watani 2011c.

19 Watani 2011d. 
politische Rolle zu überdenken. Ihm folgte Patriarch Tawāḍurūs im November 2012.

Nach dem Sturz von Ḥusnī Mubārak übernahm der Oberste Rat der Streitkräfte die Führung bis zur Wahl Muhammad Mursīs zum Präsidenten im Juni 2012. Während die Revolution einen überwiegend säkularen Charakter aufwies, begannen die islamistischen Kräfte sowohl politisch als auch diskursiv zu dominieren. Diese Kräfte bildeten keineswegs eine einheitliche Kraft, sondern standen oft in Konkurrenz zueinander. Nichtsdestotrotz erhärtete diese politische Dominanz die Angst der christlichen Ägypter vor einer Islamisierung des Landes. Der politische Arm der Muslimbruderschaft, die Partei für Freiheit und Gerechtigkeit (Hizb ul-'Adāla wa l-Ḥurrya), die salafistische al-Nur Partei (Hizb un-Nūr) und deren Allianz stellten die Mehrheit im Ober- und Unterhaus, in der verfassungsgebenden Versammlung und ein (ehemaliges) Mitglied der Muslimbruderschaft, Muhammad Mursī, gewann die Präsidentschaftswahl im Juni 2012. Die Ausarbeitung einer neuen Verfassung gestaltete sich dementsprechend schwierig und führte $\mathrm{zu}$ einer Kluft zwischen einerseits den säkularen Kräften und den kirchlichen Vertretern und andererseits den islamistischen Kräften. Im November 2012, ein paar Tage vor der Inthronisierung von Patriarch Tawāḍurūs II. traten die liberalen und kirchlichen Vertreter aus der verfassungsgebenden Versammlung aus. Daraufhin erklärte Mursī die Tätigkeit dieser Versammlung sowie sein Handeln immun gegen etwaige Klagen der Justiz, woraufhin die Proteste und die Opposition gegen die Politik von Mursī zunahmen. Im Dezember 2012 wurde die neue Verfassung mit einer geringen Wahlbeteiligung angenommen. Insgesamt brachte sie keine tiefgreifenden Veränderungen. Artikel 2 der Verfassung beinhaltete weiterhin die Prinzipien der Scharia und wurde durch einen Artikel erweitert, die Deutungshoheit über diese Prinzipien wurden vom Obersten Gerichtshof auf die Al-Azhar übertragen und die Unterstellung der nicht-Muslimen ihrem religiösen Recht bestätigt. Am 30. Juni 2013 fanden landesweite Proteste gegen Mursī statt, der am 3. Juli durch die Armee gestürzt wurde.

\section{Die Kirchen in Ägypten: der Bruch mit der Praxis von Šinūda}

Bis 2011 war die koptische Gemeinde stark durch die Persönlichkeit des koptischen-orthodoxen Patriarchen Šinūda (1923-1971-2012) geprägt. Die Revolutionsphase kann folglich als Bruch mit seinem Führungsstil und Verständnis der koptischen Gemeinde betrachtet werden. Obwohl Šinūda ein Verfechter des 
Diskurses der nationalen Einheit war, benutzten der Übergangspatriarch Bāhūumiūs und Tawāọurūs II. sowie die koptisch-katholische und die evangelische Kirchen diesen Diskurs, um differenzierte Haltungen zu untermauern.

Neben den weiter oben erläuterten externen Faktoren trugen auch die internen (koptisch-orthodoxen) Entwicklungen zur Politisierung der Kirche bei. Unter Patriarch Šinūda III. wurde die Sonntagsschulbewegung, welche in den 1920er Jahren entstand, in die Kirchenhierarchie integriert und zum Vehikel für eine Erneuerung der koptisch-orthodoxen Kirche und ihrer Gemeinde. Damit wurde das Ziel verfolgt, die Kopten zu „christianisieren“ bzw. zu „re-christianisieren“, wodurch die Kirche zum alleinigen moralischen, historischen, pädagogischen Referenzrahmen werden sollte ${ }^{20}$. Es wurden zahlreiche soziale Dienste eingeführt, und im Jahre 1980 wurde ein Bischofstum für die Jugend - das einzige seiner Art in der arabischen Welt - errichtet, um eine totale Assimilation der Jugend in die Kirche und das Eindringen in die Privatsphäre der Menschen zu ermöglichen $^{21}$. Dieses Bischofstum förderte die Gründung von christlichen Studentenverbindungen (usar) an den Universitäten ${ }^{22}$. Insgesamt nahm diese Erneuerung fundamentalistische Züge an, indem an eine vermeintliche Tradition angeknüpft wurde und ein kollektives Bewusstsein bestärkt, das auf Märtyrern und Heiligen beruhte und von griechischen, lateinischen und muslimischen Einflüssen bereinigt war ${ }^{23}$. Laure Guirguis zu Folge trug Patriarch Šinūda mit all diesen Maßnahmen zur Segregation der Kopten und zur Bildung eines Minderheitengefühls bei ${ }^{24}$. Tatsächlich ist das Bild einer bedrohten Minderheit Teil eines Diskurses, das insbesondere durch Šinūda III. nach innen gefördert wurde (nach außen förderte er den Diskurs der nationalen Einheit). Bereits im Jahre 1952 anlässlich des Brandes einer Kirche in Suez, schrieb der damalige Priester: „Der Brand ist nichts Neues für uns, unsere Geschichte [der] Verfolgung ist voll solcher Beispiele hinsichtlich Abscheulichkeit und Grausamkeit. Das Christentum in Ägypten schreitet seit dem Martyrium des Apostel Markus einen schmalen Weg über die Generationen hinweg““25.

Parallel dazu entwickelte sich die Kirche - bzw. die koptisch-orthodoxe Kirche - zum alleinigen Sprachrohr der Kopten, eine Rolle, die ihr auch das Mubārak-Regime zuschrieb. Die Unterstützung des koptisch-orthodoxen Patriarchen ging so weit, dass sich der Heilige Synod im Jahre 2005 für ein fünftes

20 El-Khawaga 1992: 47-48.

21 S.S. Hasan 2003: 184.

22 Guirguis 2012: 46.

23 Mayeur-Jaouen 2005: 363-364.

24 Guirguis 2012: 112.

25 Shenuda 2013 [1952]. 
Mandat von Mubārak aussprach und die Kopten dazu aufrief, für ihn zu wählen ${ }^{26}$. Im Gegenzug „korrigierte“ Mubārak die mangelnde politische Vertretung der Kopten, indem er koptische Politiker ins Parlament ernannte. 2010 zum Beispiel ernannte er sieben Abgeordnete ins Parlament für insgesamt 508 Sitze, von denen einige der Kirche nahestanden, andere jedoch nicht ${ }^{27}$. Die vermeintliche Loyalität der Kopten wurde jedoch nicht immer belohnt; Anlässlich der Parlamentswahlen im Jahre 2005 stellte die regierende Nationaldemokratische Partei (al-Hizb alWațanī ad-Dīmuqrāțī) nur zwei koptische Kandidaten auf ${ }^{28}$.

Folglich zeigten die Kirchen in Ägypten zuerst Ablehnung gegenüber der Protestbewegung, die am 25. Jänner 2011 begann. Erst am 15. Februar veröffentlichte die koptisch-orthodoxe Kirche und am 21. Februar die Kirchen in Ägypten eine gemeinsame Stellungnahme, in der sie ihre Unterstützung für das, was sie „die Revolution der Jugend“ nannten, äußerten ${ }^{29}$. Nach der Revolution betätigten Kirchenvertreter Äußerungen, die darauf schließen ließen, dass die Kirchen entweder ihre Haltung zur Revolution neuschrieben oder dass es Meinungsverschiedenheiten in den Kirchenhierarchien gab. Der koptisch-katholische Patriarch Anțūniyūs Nağīb sagte später „The Revolution revealed a new image of

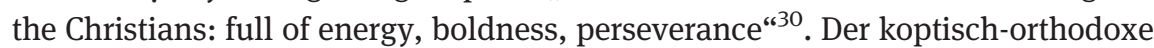
Bischof von Šubrā al-Hुayma, Bischof Murquṣ, der während der Revolution die Proteste scharf kritisierte („Wir kennen weder das Ziel dieser Demonstrationen, noch deren Details noch wer sich dahinter verbirgt“‘31), gab später gegenüber dem Autor an, dass insbesondere Patriarch Šinūda III vor der Revolution gewarnt hatte, welche die Islamisten und die Muslimbruderschaft an die Macht bringen würde, „but we said...“32.

In Folge zeigte sich nicht nur, dass die bisherige (politische) Rolle der Kirche nicht mehr aufrechtzuerhalten war, sondern dass die Haltung des Patriarchen Šinūdas als zunehmend problematisch aufgefasst wurde. Insbesondere mit der politisch aktiven koptischen Jugend vertiefte sich der Graben. In Reaktion auf das sogenannte Maspero-Massaker am 9. Oktober 2011 verdeutlichte die Stellungnahme der koptisch-orthodoxen Kirche, dass die Kirche den offiziellen Diskurs übernommen hatte; „Unser christlicher Glaube betont die Gewaltlosigkeit unter allen Umständen. So wie wir nicht vergessen, dass einige Fremde sich

26 Roussillon 2010.

27 Al-Ahram Hebdo 2010.

28 Roussillon 2010.

29 Al-Kiraza 2011a.

30 OASIS 2011: 27.

31 Proche-Orient 2011: 399.

32 Murqus 2015. 
unter unseren Söhnen gemischt haben könnten. [...]. Der heilige Synod ruft die koptische Jugend zu einem dreitätigen Fasten beginnend am Dienstag auf «33. Dieses Massaker am 9. Oktober 2011 war gewissermaßen das Endergebnis eines Konflikts um eine Kirche, der ein paar Wochen zuvor in einem Dorf in der Provinz Aswān begonnen hatte. In Reaktion auf den Brand dieser Kirche organisierten Kopten und einige Muslime Sit-ins vor dem Maspero-Gebäude. Koptische Demonstranten ignorierten die Aufforderungen der Kirche, einen Marsch zum 40. Tag des „Maspero-Massakers“ abzusagen und marschierten von der Kathedrale in al-'Abbāssiya zum Tahrir-Platz ${ }^{34}$. Als Šinūda III. dennoch Mitglieder des Militärrates zur Weihnachtsmette im Jänner 2012 einlud, riefen Kopten vor der Kathedrale „nieder mit dem Militärregime!“35

Nach dem Tode Šinūdas III. im März 2012 mehrten sich die Anzeichen für ein Überdenken der Rolle der Kirche. Anders als bisherige Untersuchungen andeuten, kam der im November 2012 gewählte Patriarch Tawāọurūs II. nicht zur bisherigen Praxis zurück. Sowohl der Übergangspratriarch Bischof Bāhūumiūs, (März-November 2012) als auch Tawāọurūs bekräftigten den Wunsch nach einer unpolitischen Kirche. Daraus wird ersichtlich, dass die Frage, inwieweit der koptisch-orthodoxe Patriarch eine politische Rolle einnimmt, auch stark von dessen Persönlichkeit abhängt. Während der Parlamentswahlen Ende 2011 soll die Kirche Berichten zufolge Anweisungen gegeben haben, für die säkular-liberale Allianz Kutla zu wählen ${ }^{36}$. Anlässlich der Präsidentschaftswahlen im Juni 2012 jedoch gab Bischof Bāhūumiūs bekannt, dass die Kirche sich gegenüber allen Kandidaten neutral verhalten und Priester bestrafen werde, die sich für einen Präsidentschaftskandidaten einsetzten ${ }^{37}$. Ein Grund dafür was sicherlich, dass Bāhūumiūs nicht über dieselbe Autorität verfügte wie Šinūda. Doch auch Tawāọurūs II. bekräftigte den Willen zu einer unpolitischen Kirchenführung. Die Kirche werde nicht mehr zwischen den Kopten und dem Staat vermitteln und er ermutigte stattdessen die Kopten, politisch und sozial engagierte Bürger zu sein: ${ }^{38}$ „The most important thing is for the church to go back and live consistently within the spiritual boundaries because this is its main work, spiritual work“39. Mehr noch, der neue Patriarch kritisierte die politische Passivität der

33 Al-Kiraza 2011b. Es gibt jedoch mehrere Berichte, die diesen Diskurs untermauern. Die unabhängige Zeitung aš-Šurūq z.B. berichtete, dass einige Demonstrierende Schusswaffen und Messer bei sich trugen (al-Shurouq 2011b).

34 Ahram Online 2012b.

35 Ahram Online 2012b.

36 Tadros 2013: 221.

37 Ahram Online 2012b.

38 Ahram Online 2012b.

39 New York Times 2012. 
koptischen Jugend ${ }^{40}$. Dass sich Tawāḍurūs II. dennoch offen gegen Präsident Muhammad Mursī äußerte und dessen Sturz auch begrüßte, wurde als nationaler Akt bezeichnet, oder wie er selber angab: „The nature of the Revolution changed, it had been robbed of its original characteristics by other religious tendencies [that] do not seem to suit Egypt as a whole“ ${ }^{441}$. Anders als Šinūda III. prangerte Tawādurūs offen die autoritären Ausuferungen von Mursī an. Wie die Haltung Tawāḍurūs zu deuten ist und ob sie tatsächlich einen Bruch mit Šinūda darstellte, sei dahingestellt. Hervorzuheben ist jedoch, dass die beiden Patriarchen sich unterschiedlicher Druckmittel bedienten, um ihren Unmut auszudrücken. Patriarch Šinūda III. etwa hatte Methoden entwickelt, um Druck auf die Regierung auszuüben, welche stark religiöse Symbole einbezogen. Zum Beispiel in Folge schwerer Auseinandersetzungen zwischen koptischen Demonstranten und der Polizei um den Bau einer Kirche in al-'Umrāniyya im November 2010, zog sich Šinūda III. in die Klöster des Wādī al-Națūn zurück, um die Freilassung von 133 inhaftierten Kopten zu erwirken ${ }^{42}$. Er rief auch zu Fasten und Gebeten auf, etwa nach Konflikten in Alexandria 2005 ${ }^{43}$. Tawāḍurūs II. hingegen bediente sich zwar des Diskurses der nationalen Einheit, untermauerten diesen aber durch die Verwendung allgemeiner Medien und moderner Kommunikationsmittel wie eine im Fernsehen live-übertragene Rede am 8. April $2013^{44}$ oder verschickte Nachrichten über Twitter ${ }^{45}$ am 29. Juni 2013, um die Kopten dazu aufzurufen, sich am Erhalt des nationalen Wohls zu beteiligen: „Egypt needs every Egyptian today! We must think, discuss, and express our desire for our nation together without violence, enmity or bloodshed“, „The national responsibility of the Coptic Church of Egypt demands us all to support the measures that protect our country and achieve our freedom without violence or recklessness. Long live Egypt, safe and secure“ ${ }^{46}$.

Der Kontrast zum Führungsstil und Diskurs Šinūdas wird umso deutlicher, wenn man die Haltung der koptisch-katholischen und der koptisch-evangelischen Kirchen in dieser Zeit betrachtet, Ein Vergleich zwischen al-Kirāza und der koptisch-katholischen Zeitung aṣ-Șalāḥ ist hier sehr aufschlussreich. Während Papst Šinūda in der von ihm verfassten Kirchenzeitung al-Kirāza die Vorfälle gegen Kopten aufzählte und den baldigen Machtantritt der Islamisten

40 Proche-Orient Chrétien 2013b: 340.

41 OASIS 2013: 82.

42 Al-Ahram Hebdo 2010.

43 Shenoda 2007: 601.

44 Guardian 2013.

45 Eine der ersten Entscheidungen der Heiligen Synode unter Tawāḍuūus war die Errichtung eines Facebook- und Twitter-Accounts (Proche-Orient Chrétien 2013a: 126).

46 Van Doorn-Harder 2013. 
ankündigte ${ }^{47}$, übten zwei Artikel in aș-Ṣalāh Kritik an den ihrer Meinung nach falschen Zahlen zur koptischen Emigration und an das, was sie die „Produktion der Angst“ nannten, welche das Ziel verfolge, Ägypten von seinen Kopten zu leeren. ${ }^{48}$ In dieser Zeitschrift sprach die koptisch-katholische Kirche auch von einem Zustand der „Phobie“ (fūbyā), den die Kopten gegenüber der Muslimbruderschaft hätte und welche ihrer Meinung nach unbegründet $\operatorname{sei}^{49}$. Ein weiterer Unterschied war diese Haltung gegenüber der Muslimbruderschaft, da sowohl die koptisch-katholische als auch die koptisch-evangelische Kirchen aktiv den Dialog mit der Organisation suchten. Im März 2012 unterzeichnete die Muslimbruderschaft mit den Vertretern der koptisch-evangelischen Kirche ein Dokument zur Anerkennung dergleichen Rechte und Pflichten für alle Ägypter ${ }^{50}$. Zwischen 2011-2013 gab es jedoch zahlreiche Kontakte zwischen dem koptisch-orthodoxen Klerus und Vertretern der Muslimbruderschaft (auch Gouverneure und Partei Freiheit und Gerechtigkeit), etwa im Kontext der Kontroverse um den islamkritischen Film „The Innocence of Muslims“. Der Unterschied ist jedoch, dass die koptisch-evangelische und die koptisch-katholische Kirchen offiziell keine Vorbehalte zeigten, während sich die koptischorthodoxe Kirche an die politischen Umstände anpasste. Ein weiterer nennenswerter Aspekt ist das Verständnis des Begriffes „Kopten“. Die koptisch-katholische Kirche propagierte nämlich die Ansicht, dass sie keine Minderheit in einem ethnischen ('araqī), konfessionellen (ță'ifi) und auch nicht in einem religiösen Sinn bildeten ${ }^{51}$. Tatsächlich verwendete die koptisch-katholische Kirche in aṣ-Ṣalāh selten den Begriff „aqbāt" („Kopten“), sondern eher „masīhiyyūn“ („Christen“). Der Begriff „aqbāt" hat eine starke ägyptische und ethnische Konnotation, welcher mit dem Diskurs der nationalen Einheit der koptisch-orthodoxen Führung, allen Vorrang Šinūda, ermöglicht(e), die Verwurzelung der Christen in und Zugehörigkeit zu Ägypten zu untermauern. Dieser Diskurs bedient sich oft einer bildreichen Sprache. Bischof Mūsā für die Jugend sagte anlässlich der Revolution: „Alle tragen dieselben Gesichtszüge, auf denen Ägypten geschrieben ist“"52.

Dieser zweite Teil hat bereits aufgezeigt, wie die unterschiedliche Handhabung des Diskurses der nationalen Einheit eigentlich auf unterschiedliche Haltungen und Mittel hindeuten. Es ist insbesondere hervorzuheben, dass eine

47 Al-Kiraza 2011a.

48 Al-Salah 2012a.

49 Al-Salah 2012b: 31.

50 Facebook 2012.

51 Al-Salah 2012b: 32-33.

52 Watani 2011b. 
Verbesserung der Lage „der Kopten“ für die koptisch-orthodoxe Kirche über einen Diskurs der nationalen Einheit führt, der systematisch zwischen Kopten und Muslimen unterscheidet, für die koptisch-katholische Kirche hingegen über einen Diskurs, der gerade die Unterscheidung herunterspielt.

\section{Der Koptische Aktivismus}

Dieser dritte Teil wird anhand des koptischen Aktivismus, welcher am Vorabend der Revolution bereits begonnen hatte, sich von der politischen Bevormundung durch die Kirche zu emanzipieren, verstärkt aufzeigen, wie der Diskurs der nationalen Einheit verwendet wurde. Dabei ist $\mathrm{zu}$ beachten, dass sich dieser Diskurs der Einheit und der verstärkte Konfessionalismus nicht notwendigerweise ausschließen müssen.

Eines der prägnantesten Momente des „Arabischen Frühlings“ war die Verwendung christlicher Symbole zusammen mit muslimischen Symbolen am Tahrir Platz, welche die nationale Einheit und die Teilnahme der Kopten materialisierte. Im Zuge des Arabischen Frühlings traten verstärkt Entwicklungen $\mathrm{zu}$ Tage, die bereits vor 2011 begonnen hatten und eine zunehmende Auflehnung gegen die politische Bevormundung durch die Kirche verdeutlichte und die Diskriminierungen gegenüber Kopten eintraten. Ein erster Wendepunkt gegen diese Bevormundung hatte nach dem Angriff auf die Weihnachtsmette in Nağ Hammadī, Oberägypten, im Jänner 2010, bei der sechs Menschen erschossen wurden, stattgefunden, als Kopten erstmals außerhalb der Kirche vor staatlichen und zivilen Einrichtungen demonstrierten. In Reaktion darauf, organisierte Hānī al-Ğazīrī, eine wichtige Figur dieses aufkeimenden koptischen Aktivismus, am 14. Februar 2010 einen Protest am Tahrir-Platz mit der Begründung: „Ich bin Ägypter, ich habe ein Recht dazu“53. Ein weiterer Wendepunkt waren die bereits erwähnten gewalttätigen Auseinandersetzungen zwischen koptischen Demonstranten und der Polizei um den Bau einer Kirche in al'Umrāniyya im November 2010, in Folge dessen die koptischen Demonstranten den Sitz des Gouverneurs von al-Ǧìza stürmten ${ }^{54}$. Mariz Tadros weist auf die Bedeutung von al-'Umrāniyya hin, weil zum ersten Mal nicht die koptische Elite den Protest anführte, sondern Durchschnittsbürger ${ }^{55}$. Nach dem Anschlag in Alexandria am 6. Jänner 2011 fanden Demonstrationen von Seiten der Kopten

53 Al-Gazeri 2015.

54 Al-Ahram Hebdo 2010.

55 Tadros 2013: 107. 
statt, die sich sowohl gegen den Staat als auch gegen die Kirchenführung richteten. Jugendliche unterbrachen einen Vertreter der Kirche, als dieser sich bei Ḥusnī Mubārak bedanken wollte ${ }^{56}$. Nach einer Pressekonferenz mit Patriarch Šinūda wurden die Autos des Shayh al-Azhar, des Muftis und des Ministers für fromme Stiftungen mit Steinen beworfen ${ }^{57}$. Im ganzen Land fanden Demonstrationen gegen den Anschlag statt, an der sowohl Muslime als auch Kopten teilnahmen ${ }^{58}$.

Auch nach der Revolution erfuhr diese Emanzipation weitere Entwicklungsphasen. In Reaktion auf die anhaltenden Angriffe auf Kopten verwandelten koptische Aktivisten im März 2011 den Maspero-Platz vor dem Sitz des Staatsfernsehens in Kairo in einen alternativen Tahrir-Platz für spezifisch koptische Anliegen. Eine wichtige Entwicklung nach der Revolution war die schrittweise Institutionalisierung des koptischen Aktivismus und die Proliferation koptischer (Jugend)Organisationen. Nennenswert ist, dass ein großer Teil des koptischer Aktivismus unstrukturiert blieb und spontan, meistens in Reaktion auf Fällen von Gewalt gegen Kopten zu Tage trat. Ein wichtiger Wegbereiter dieses neuen strukturierten koptischen Aktivismus war Hānī al-Ğazīrī, der sich in den 2000er Jahren der Kefaya Bewegung (Harakat Kifāya), die eine fünfte Amtszeit von Ḥusnī Mubārak und die Nachfolge dessen Sohnes Ğamāl ablehnte, angeschlossen hatte und sich in den folgenden Jahren von einem allgemeinen, zivilrechtlichen Engagement in eine dezidiert konfessionelle Richtung orientierte. Im Jahre 2009 gründete er die Organisation „Kopten für Ägypten“ (Aqbāt min Ağl Mișr) mit dem Ziel, die Aufmerksamkeit des Staates auf koptische Belange zu richten $^{59}$, die nach sogenannten religiösen Konflikten eingesetzt wurden. Diese konfessionelle Richtung wurde auch vom einem guten Teil der Tahrir-Aktivisten eingeschlagen. So entstanden nach der Revolution von 2011 eine Reihe von koptischen Jugendorganisation, wie die Koalition der Kopten Ägyptens (I'tilāf Aqbāt Miṣr), die Union der Maspero-Jugend (Ittihāa Šabāb Māsbīrū), die Bewegung Kopten ohne Beschränkungen (Harakat Aqbāṭ bilā Quyūd), die „Christlichen Brüder“ (al-Ihwwān al-Masīhiyyūn), Letztere in Reaktion auf die Wahl von Muhammad Mursī ${ }^{60}$. Die Union der Maspero-Jugend wurde im März 2011 nach der Vertreibung von Kopten aus dem Dorf 'Ațịh in der Provinz Gizeh gegrün$\operatorname{det}^{61}$, wurde aber nach dem Maspero-Massaker von den Familien der Opfer

56 Ahram Online 2012b.

57 BBC Jänner 2011.

58 Watani 2011a.

59 Al-Gaziri 2015.

60 Al-Ahram Hebdo 2012b.

61 Magdi 2015. 
scharf kritisiert und der Korruption bezichtigt ${ }^{62}$. Diese Organisation war vor allem sehr medienpräsent. Insgesamt bezeugten die Namen dieser Organisationen ein klares Bekenntnis zur „koptischen Identität“. Andere Organisationen, die in dieser Zeit aktiv waren, erfuhren weitaus weniger Beachtung, wie z.B. die 2011 gegründete Jugendorganisation „Bewegung der Zivilrechte für Christen“ (Harakat ul-Huqūq ul-Madaniyya li l-Masīhiyyīn) ${ }^{63}$. Weitere Gründe für die erhöhte Sichtbarkeit einiger Organisationen gegenüber anderen war die stärkere Mitgliedzahl und die größere Mobilisierungskraft; „Wir verwenden politische Mittel wie die Medien, Sit-ins Märsche“64. Diese Mobilisierungskapazität zeigte sich anlässlich der Proteste vor der amerikanischen Botschaft in Kairo im September 2011 gegen das islamkritische Video „The Innocence of Muslims“, an der die Union der Maspero-Jugend und die Koalition der Kopten Ägyptens, aber nicht die Christlichen Brüder und Hānī al-Ğazīrī teilnahmen ${ }^{65}$.

Auch wenn diese Organisationen auf den ersten Blick einen dezidiert konfessionellen Charakter aufwiesen (und in der Literatur oft im Zusammenhang mit religiösen Konflikt gebracht werden), stellt sich die Frage, wie sich dies verdeutlichte und die Hypothese, dass diese konfessionelle Ausrichtung nicht nur dem Geist der Zeit entsprach, sondern auch mehrere Funktionen erfüllte. Der Umgang mit der koptischen Identität verdeutlichte einen Generationsbruch zwischen der älteren, bereits politisch aktiven, Generation und einer jüngeren Generation, die zum Großteil am Tahrir-Platz erste politische und zivilrechtliche Erfahrungen sammelte. Akteure wie der ehemalige Abgeordnete Ğamāl As'ad sahen in der Gründung der Organisation „die Christlichen Bruder“ einen gefährlichen Schritt: „Une telle association n'est sûrement pas dans l'intérêt des coptes, au contraire elle fait l'affaire des islamistes radicaux qui prônent un État religieux [...] si les coptes aspirent à un État civil basé sur la citoyenneté, il faut qu'ils se considèrent comme Égyptiens non comme une minorité chrétienne.“66 Schon 2005 hatte Ğamāl As'ad die Selbstbehauptung Šinūdas als politisches Sprachrohr der Kopten angeprangert; Denn dies würde bedeuten, die Kopten bildeten „un bloc homogène séparé de la trame nationale“67. Somit wird deutlich, dass koptische Persönlichkeiten wie Ğamāl As'ad die Existenz einer separaten, selbstständigen koptischen Identität ablehnten, während dies wie selbstverständlich von den jungen Aktivisten angenommen wurde. Dies ist

62 Daily News Egypt 2012.

63 Facebook 2011a.

64 Magdi 2015. Weder Fādī Yūsif noch Mīnā Magdī nannten im Jänner 2015 genaue Zahlen.

65 Al-Yawm al-Sabi 2012.

66 Al-Ahram Hebdo 2012a.

67 Roussillon 2010. 
das Ergebnis einer jungen Generation von Kopten, die in der Kirche aufgewachsen ist und das Ergebnis des Charakters dieser Erneuerung selber. Mīnā Magdī, Koordinator der Union der Maspero-Jugend, rechtfertigte die Errichtung einer spezifisch koptischen Organisation damit, dass bürgerrechtliche Bewegungen sich aus den betroffenen Gruppen zusammensetzen: "must ha[ve] people to speak about them, to defend their case and they must be from this group itself, [...] "we are not a religious group, we are a political group" und wies darauf hin, dass der Leiter des Politikbüros koptisch-katholisch war ${ }^{68}$.

Dieser konfessionelle Charakter artikulierte sich nicht im Gegensatz zu, sondern vielmehr in Kontinuität mit dem Diskurs der nationalen Einheit. Dies erklärt vielleicht, warum nie eine spezifisch koptische Organisation gegründet wurde. Diese Generation der koptischen Aktivisten wurde zwar mit einer genuinen koptischen Identität sozialisiert, aber auch mit dem Diskurs der nationalen Einheit: „it supports the one nation“, sagte Mīnā Magdīí ${ }^{69}$. Dieser Diskurs sollte jedoch nicht als Floskel betrachtet werden. Der Diskurs der nationalen Einheit wurde wiederkehrend verwendet, um Missstände anzuprangern. Im September 2012 verurteilte die Bewegung „Kopten ohne Beschränkungen“ das islamkritische Video „The Innocence of Muslims“, übte aber gleichzeitig auch scharfe Kritik an der vermeintlichen Doppelmoral der Medien, des Staates und der Gesellschaft im Allgemeinen, wenn christliche Symbole und Personen angegriffen werden. Diese Kritik wurde durch einen Hinweis auf die nationale Einheit untermauert $^{70}$.

Diese Gratwanderung zwischen Konfessionalismus, offener Kritik und Kontinuität zum Diskurs der nationalen Einheit erfordert, dies in einem breiteren Kontext zu betrachten, der über die Thematik der „fitna țā'ifiyya“ hinausgeht und die Interaktion mit dem Staat und den islamistischen Kräften einbezieht.

\section{Der koptische Aktivismus, der Staat, die Islamisten: zwischen Polemik und Pragmatismus}

Der dritte Teil umfasst die Frage der Kopten im Zusammenhang mit „dem Staat“ und den islamistischen Kräften. Dadurch, dass die Muslimbruderschaft an die Macht kam, bezieht sich dieser Teil nicht nur auf die bisher erwähnten Akteure

68 Magdi 2015.

69 Magdi 2015.

70 Al-Balad 2012. 
des koptischen Aktivismus, sondern auch auf die christlichen Ägypter im Allgemeinen.

Ein erster wichtiger Aspekt ist die Kontinuität staatlicher Praktiken und Haltungen im Zusammenhang mit der sogenannten „Koptenfrage“, sei es unter Mubārak, dem Obersten Rat der Streitkräfte oder Muhammad Mursī. Im Kontext anhaltenden „interkonfessionellen Zwists“ wurden weiterhin Aussöhnungsversammlungen oktroyiert statt strafrechtlicher Verfolgung, und die neuen Akteure des politischen Lebens, wie Salafisten einbezogen. In Folge gewalttätiger Auseinandersetzungen in einem Dorf in der Provinz Ḥilwān im März 2011 stellte die Armee eine Delegation bestehend aus dem berühmten salafistischen Predigern Muhammad Hassān and Șafwat Hiğāzī, die beide brennende Reden für die nationale Einheit lieferten, und liberalen Politikern wie 'Amr Hamzāwī und George İṣhāq zusammen. In diesem Zusammenhang ist das scheinbare Versagen des Staates, Recht und Ordnung aufrecht zu erhalten erwähnenswert. Die Regierung gab z.B. anlässlich anhaltender Proteste gegen die Nominierung eines koptischen Gouverneurs für die Provinz Qinā schließlich nach ${ }^{71}$. Die Schwäche des Staates wurde auch im Falle der gewalttätigen Auseinandersetzungen im Stadtviertel Imbāba in Gizeh im Mai 2011 vor allem hinsichtlich des Versagens, Schutz zu gewähren, deutlich. In diesem Fall griffen die Sicherheitskräfte erst drei Stunden nachdem Salafisten angefangen hatten, eine Kirche und koptisches Eigentum anzugreifen, ein ${ }^{72}$. Eine weitere Praxis war die Ernennung koptischer Akteure ins Parlament, um die politische Unterrepräsentierung der Kopten zu „korrigieren“, wie etwa im Zuge der Parlamentswahlen, als Ṭanțāwī vier Kopten ernannte $^{73}$. Zudem setzte Muhammad Mursī mit alten Praktiken fort, wie etwa die Ernennung eines koptischen Beraters in seine Regierung, Samīr Murquṣ, und die Übernahme des Diskurses der nationalen Einheit. Ebenso wie der Staat unter Husnī Mubārak, betrachtete die Muslimbruderschaft die Kopten als homogene Minderheit, die politisch durch die Kirche vertreten wird und nicht in erster Linie als Bürger. Diese Haltung wurde im Allgemeinen von allen islamistischen Kräften eingenommen. Dies zeigte sich in zwei Fällen, als die Organisation einen Dialog mit der koptischen Jugend initiieren wollte, sich dafür aber an die Kirche wandte $^{74}$.

Zwei gegensätzliche aber nennenswerte Entwicklungen zwischen 2011 und 2013 waren einerseits die zunehmende Polarisierung und Polemik zwischen Christen und Muslimen und andererseits die unwahrscheinliche Interaktion.

\footnotetext{
71 Sidhom 2011.

72 Watani 2011f.

73 Proche-Orient Chrétien 2012: 331.

74 Watani 2011e.
} 
Im Zuge der Revolution von 2011 traten Diskurse in den Vordergrund, die bisher marginalisiert waren, wie jener der salafistischen Kräfte, aber auch koptische Diskurse, wie weiter oben erwähnt. Die koptischen Jugendorganisationen waren viel vehementer und offener in ihrer Kritik als die ältere, da sie nicht davor zurückscheuten, lokale Imame und bekannte salafistische Prediger offen für ihre vermeintliche Verantwortung in interkonfessionellen Spannungen anzuprangern. Mitglieder der Union der Maspero-Jugend machten den Gouverneur von Aswān sowie Vertreter der salafistischen Bewegung für den Konflikt verantwortlich, der schließlich zum Maspero-Massaker führte ${ }^{75}$. Umgekehrt nahmen politische Diskurse von islamistischen und salafistischen Akteuren gegenüber Christen nicht notwendigerweise $\mathrm{zu}$, sondern wurden sichtbarer, vor allem in allgemeinen ägyptischen Medien berichtet. In diesem Diskurs gelten Christen vermehrt als Polytheisten und Ungläubige und nicht mehr als die vom Islam anerkannten ahl ul-kitāb. Insbesondere anlässlich der Weihnachtsfeierlichkeiten im Jänner 2011 schlugen die Nur Partei und die ă̆-Ğamā'a al-Islāmiyya die Einladung Šinūdas aus mit der Begründung, sie würden dadurch den Kult der drei Götter billigen ${ }^{76}$. Zusätzlich zu dieser dogmatischen Grundhaltung war die Haltung der Islamisten auch von den wahrgenommenen Handlungen der Kopten beeinflusst - so wie umgekehrt die koptischen Aktivisten durch ihre Wahrnehmung der Islamisten. Dieser junge koptische Aktivismus, der offen Zustände anprangerte und mobilisierte, sorgte für großen Unmut in salafistischen Kreisen. Anlässlich des Maspero-Massakers bekundeten zwar salafistische Akteure wie Yāsir Burhāmī, Anführer der ad-Da'wa as-Salafiyya, oder die Salafistische Front (ağ-Ğabha as-Salafiyya) ihre Trauer, sie machten aber die koptischen Aktivisten am Maspero-Platz für das Massaker verantwortlich und betrachteten sie als Gefahr für die Stabilität Ägyptens ${ }^{77}$. Im Kontext der zunehmenden Polarisierung, vor allem des Präsidentendekrets im November 2012, nahmen auch von Seiten der Muslimbruderschaft die verbalen Attacken gegen die Kopten zu. Muhammad al-Biltāğī und Șafwat Ḥiğāzī, letzterer steht der Muslimbruderschaft nahe, deuteten in ihren Auftritten auf TV Misr 25 und in Kundgebungen an, die Kopten würden eine fünfte Kolonne bilden und die Kirche wäre in einer Destabilisierungskampagne gegen die Regierung involviert ${ }^{78}$.

Der von der Revolution eingeleitete Demokratisierungsprozess bedeutete vor allem in seiner Anfangsphase die Möglichkeit für zahlreiche islamistische und salafistische Akteure, ihre politischen Ziele $\mathrm{zu}$ erreichen und in diesem

75 Al-Shuruq 2011a.

76 Ahram Hebdo 2012a.

77 Siehe Facebook 2011b.

78 Paul Sedra 2012b. 
Zusammenhang stellten „die Kopten“ einen hohen symbolischen Wert dar, um sich als politisch seriöse und inklusive Akteure zu etablieren. Dies betraf sowohl die Muslimbruderschaft, die al-Nur Partei, als auch den weiten oben erwähnten salafistischen Prediger Muhammad Hassān und sogar die Salafistische Front. Sowohl die Partei für Freiheit und Gerechtigkeit als auch die Hizb un-Nūr behaupteten, dass sich christliche Ägypter unter den Gründungsmitgliedern und den Mitgliedern befanden. Mariz Tadros Angaben zufolge sollen es 23 Kopten statt der angegebenen 93 in der Partei für Freiheit und Gerechtigkeit und fünf Kopten statt der von der Nur Partei angegebenen 50 Kopten gewesen sein ${ }^{79}$. Ein prominentes Mitglied der Partei für Freiheit und Gerechtigkeit war dessen ehemaliger Vizegeneralsekretär und koptisch-evangelische Intellektuelle Rafīq Habīb, der im Dezember 2012 in Folge des kontroversen Dekrets von Mursī zurücktrat. Der ehemalige Generalsekretär der Hizb un-Nūr, 'Imād 'Abd al-Ghaffūr, gab an, dass weniger Kopten als er erhofft hatte, der Partei beitraten ${ }^{80}$. Wie relevant die Kopten waren, zeigte sich auch am Beispiel der Konferenzgründung der salafistischen Volkspartei (Hizb uš-Ša $b$ ) bei der auch die kleine, kontroverse koptische Organisation „Kopten von 38“ (Aqbāt 38) ${ }^{81}$ anwesend war ${ }^{82}$. Dieses Beispiel zeigt auch, wie umgekehrt „Muslime“ und sogar islamistische Kräfte für die koptischen Aktivisten wichtig sein konnten, um ihre Anliegen zu legitimieren. Ebenso wies der Vorsitzende der Koalition der Kopten Ägyptens, Fādī Yūsif, darauf hin, dass die Organisation bedeutende Muslime, darunter einen Azhari-Gelehrten zählte ${ }^{83}$.

Daraus wird ersichtlich, dass auch islamitische Akteure sich der Bedeutung und Dominanz des Diskurses der nationalen Einheit, welcher eine moderate Haltung gegenüber den Kopten erforderte, bewusst waren.

\section{Conclusio}

Viele der hier beschriebenen Dynamiken schwanden mit dem Machtantritt asSīsīs in Juli 2013 ab. Die meisten der hier erwähnten koptischen Organisationen

79 Tadros 2013: 211.

80 YouTube 2012.

81 Genannt in Anlehnung an das koptisch-orthodoxe Scheidungsgesetz von 1938, forderte die Gruppe „Kopten von 38“ eine Wiedereinführung der Scheidung deren Bedingungen unter Šìnūda III. Anfang der 1970er Jahren verschärft wurden. Somit kann eine koptisch-orthodoxe Person noch immer vor einem zivilen Gericht eine Scheidung (welches das Gesetz von 1938 weiterhin anwendet) erwirken, diese wird aber von der Kirche nicht anerkannt und eine Wiederheirat z.B. ist nicht möglich.

82 Ahram Online 2012a.

83 Youssef 2015. 
haben an Bedeutung verloren. In einem Gespräch mit dem Autor in Jänner 2015 befand Hānī al-Ğazīrī die Aufrechterhaltung solcher dezidiert koptische Organisation gewissermaßen schädlich für die Zukunft Ägyptens; „Die Konzepte „Christ“ und „Muslim“ müssen unbedingt vom Gedächtnis der Menschen getilgt werden; Es wird nur „Ägypter“ übrigbleiben. Ich bin Ägypter“84.

In diesem Beitrag wurden die der koptischen Gemeinde internen und externen Faktoren aufgezeigt, die jeweils vor und nach dem Arabischen Frühling die Rolle der Kirche und die Rahmenbedingungen für das politische Engagement der Kopten definierten. Wichtige Faktoren waren die sozialen, politischen und demographischen Umwälzungen der letzten Jahrzehnte, die Haltung des Staates und der islamistischen Kräfte, insbesondere der Muslimbruderschaft, sowie jener der Kirchenführung. Im Falle des Staates wurden dessen Durchsetzungsschwäche und Handhabung mit religiösen Konflikten verdeutlicht. Der Staat betrachtete auch nach der Revolution, sei es das Militärregime oder Muhammad Mursī, die Kopten als homogene Minderheit und nicht als selbstständige Bürger. Es wurde jedoch auch aufgezeigt, welche Bedeutung einzelnen Persönlichkeiten wie Šinūda III., Übergangspatriarch Bāhūmiūs und Tawāḍurūs II. bei der Definition der Rolle der Kirche und vor allem ihrer Mittel (die christlichen Symbole vs. die nationalen Referenzen) zukam. Somit wurde insgesamt das Wechselspiel zwischen strukturellen Kontinuitäten (die Haltung des Staates) und die Spielräume einzelner Patriarchen deutlich.

Während die ältere Generation an einem säkularen Rahmen festhielt, brachte ein Teil der jungen koptischen Aktivisten eine konfessionelle Dimension ein. Obwohl diese christliche Identität im Vordergrund stand, bedienten sich die jungen koptischen Aktivisten zivilrechtlicher Mittel und nicht religiöser Symbole (Sit-in, Proteste, moderne Kommunikationsmittel). Zusätzlich wurde der Fokus auf Akteure gelegt, die oft wenig Beachtung finden - die koptisch-katholische Kirche, die evangelische Kirche, andere koptische Jugendorganisationen als die Union der Maspero-Jugend - sowie die Interaktion mit muslimischen und islamistischen Akteuren.

Dieser Beitrag nahm Halbwachs Theorie des kollektiven Gedächtnisses - in diesem Fall den Diskurs der nationalen Einheit - als Ausgangspunkt, um die Gratwanderung der Kopten nach der Revolution zwischen politischer Emanzipation und Minderheitenstatus - also als Objekte diverser Diskurse - zu ergründen. In diesem Zusammenhang erwies sich dieser Diskurs der nationalen Einheit in seiner Unterscheidung zwischen Kopten und Muslimen als besonders nützliches theoretisches Rahmen, um unterschiedliche Haltungen, Interesse und Vorgehensweisen vielfältiger Akteure $\mathrm{zu}$ erfassen. Statt diesen Diskurs als

84 Al-Gazeri 2015. 
Floskel zu betrachten, welcher dazu diene, von der Gewalt gegen Kopten abzulenken ${ }^{85}$, ergab dieser Beitrag, dass er sehr wohl als Mittel verwendet wurde, um Kritik an der Gewalt gegen Kopten auszuüben, um koptische Anliegen zu legitimieren, um als moderate politische Akteure aufzutreten (im Falle der islamistischen Akteure). Dieser Ansatz ermöglichte es, über die anhaltenden Gewaltausbrüche auch 2011 hinauszugehen, und die vielfältigen und widersprüchlichen Dynamiken aufzuzeigen.

Acknowledgements: Ich möchte mich insbesondere beim Österreichischen Austauschdienst für die Zuerkennung des Marietta Blau-Stipendiums zum Zwecke eines Forschungsaufenthaltes in Kairo in 2015 bedanken. Mein Dank gebührt auch dem Department of Near Eastern Studies/Transregional Institute an der Universität Princeton.

\section{Bibliographie}

Al-Ahram Hebdo (2010): „Encourager l'esprit de citoyenneté“. 22.-28. Dezember, Jahr 17. Nummer 850: 3-4.

Al-Ahram Hebdo (2012a) : „Prêtres, généraux et barbus“. 11-17. Jänner. Jahr 18. Nummer 904: 7. Al-Ahram Hebdo (2012b): „Une démarche symbolique“. 18-24. Juli, Jahr 18. Nummer 931: 8. Ahram Online (2012a): „Egypt's Salafist Front launches 'People Party'“. 20. Oktober. http:// english.ahram.org.eg/NewsContent/1/64/56087/Egypt/Politics-/Egypts-Salafist-Frontlaunches-People-Party.aspx. (17. Jänner 2017).

Ahram Online (2012b): „Church-state relations yet to change in Morsi's Egypt“. 18. November. http://english.ahram.org.eg/NewsContent/1/64/58089/Egypt/Politics-/-Churchstate-rela tions-yet-to-change-in-Morsis-Egy.aspx. (17 Jänner 2017).

Armanios, Febe (2011): Coptic Christianity in Ottoman Egypt. New York: Oxford University Press. BBC Arabic (2011): مصر: متظاهرون أقباط يهاجمون سيارات وفد شيخ الأزري :Ägypten: koptische Demonstranten greifen die Autos der Delegation des Šayb Ul-Azhar an)“. 2. Jänner. http://www. bbc.com/arabic/middleeast/2011/01/110102_egypt_alex_church_attack.shtml. (17 Jänner 2017).

Al-Balad (2012): , حركة " أقباط بلا قيود" نرفض الإساءة للمقسات الإسلامية .. ونطالب بوضع حد للإساءات لرموزنا .المسيحية. (Die Bewegung „Kopten ohne Beschränkungen“: Wir lehnen die Verletzung islamischer Heiligtümer $\mathrm{ab}$... und fordern eine Grenzsetzung für die Verletzung unserer christlichen Symbole)“. 10. September. http://www.el-balad.com/261182 (6. Juli 2017).

Daily News Egypt (2012): „Families of Maspero Victims attack Maspero Youth Union“. 6. Oktober. http://www.dailynewsegypt.com/2012/10/06/families-of-maspero-victimsattack-maspero-youth-union/. (17 Jänner 2017).

Davis, Stephen J. (2008): Coptic Christology in Practice: Incarnation and Divine Participation in Late Antique and Medieval Egypt. Oxford; New York: Oxford University Press.

85 Siehe Guirguis 2012: 42-44. 
Elsässer, Sebastian (2013): „Kreuz und Halbmond wieder vereint? Revolutionäre Solidarität und religiöse Spannungen während und nach der ägyptischen Revolution“. In: Revolution und Regimewandel in Ägypten. Hrsg von Holger Albrecht und Thomas Demmelhuber. Baden: Nomos, 257-280.

Elsässer, Sebastian (2014): The Coptic Question in the Mubarak Era. New York: Oxford University Press.

Facebook (2011a): حركة الحقوق المدنية للمسيحيين في مصر/. (Bewegung der Zivilrechte für Christen)“ https://ar-ar.facebook.com/cecrm/info/?tab = page_info (6. Juli 2017).

Facebook (2011b): ,بيان الجبهة السلفية بخصوص أحداث ماسبيرو/. (Stellungnahme der Salafistischen Front betreffend die Ereignisse von Maspero)“ 9. Oktober. https://es-la.facebook.com/notes/\% D9\%85\%D8\%A8\%D8\%A7\%D8\%B4\%D8\%B1-6-\%D8\%A5\%D8\%A8\%D8\%B1\%D9\%8A\%D9\% 84/\%D8\%A8\%D9\%8A\%D8\%A7\%D9\%86-\%D8\%A7\%D9\%84\%D8\%AC\%D8\%A8\%D9\%87\% D8\%A9-\%D8\%A7\%D9\%84\%D8\%B3\%D9\%84\%D9\%81\%D9\%8A\%D8\%A9-\%D8\%A8\%D8\% AE\%D8\%B5\%D9\%88\%D8\%B5-\%D8\%A3\%D8\%AD\%D8\%AF\%D8\%A7\%D8\%AB-\%D9\%85\% D8\%A7\%D8\%B3\%D8\%A8\%D9\%8A\%D8\%B1\%D9\%88/171970539555285/ (6. Juli 2017).

Facebook (2012): بيان عن اللقاء المشترك للإخوان المسلمين وقيادات الكنيسة الإنجيلية (Stellungnahme des Treffens zwischen den Muslimbrüdern und den Anführern der evangelischen Kirche)“. 28. Februar. https://ar-ar.facebook.com/notes/\%D9\%85\%D8\%B5\%D8\%B1-\%D9\%84\% D9\%84\%D9\%85\%D8\%B3\%D9\%8A\%D8\%AD/\%D8\%A8\%D9\%8A\%D8\%A7\%D9\%86-\% D8\%B9\%D9\%86-\%D8\%A7\%D9\%84\%D9\%84\%D9\%82\%D8\%A7\%D8\%A1-\%D8\%A7\% D9\%84\%D9\%85\%D8\%B4\%D8\%AA\%D8\%B1\%D9\%83-\%D9\%84\%D9\%84\%D8\%A5\%D8\% AE\%D9\%88\%D8\%A7\%D9\%86-\%D8\%A7\%D9\%84\%D9\%85\%D8\%B3\%D9\%84\%D9\%85\% D9\%8A\%D9\%86-\%D9\%88\%D9\%82\%D9\%8A\%D8\%A7\%D8\%AF\%D8\%A7\%D8\%AA-\% D8\%A7\%D9\%84\%D9\%83\%D9\%86\%D9\%8A\%D8\%B3\%D8\%A9-\%D8\%A7\%D9\%84\% D8\%A5\%D9\%86\%D8\%AC\%D9\%8A\%D9\%84\%D9\%8A\%D8\%A9/399112776772681/. (17 Jänner 2017).

Al-Gaziri, Hani (2015): 23. Jänner. Persönliches Interview. Kairo.

Guardian (2013): „Egypt’s Coptic Pope criticises Islamist President over Sectarian Violence“. 9. April. http://www.theguardian.com/world/2013/apr/09/egypt-coptic-pope-sectarian-vio lence. (17. Jänner 2017).

Guirguis, Laure (2012): Les Coptes d'Égypte: Violences communautaires et transformations politiques (2005-2012). Paris: Karthala.

Halbwachs, Maurice (1980 [1950]): The Collective Memory. Übers. von Francis J. Ditter und Vida Yazdi Ditter. New York: Harper colophon books.

Hasan, Sana S. (2003): Christians vs. Muslims in Modern Egypt. The Century-Long Struggle for Coptic Equality. New York: Oxford University Press.

Ibrahim, Vivian (2013): The Copts of Egypt. The Challenges of Modernisation and Identity. London: I.B. Tauris.

Iskander, Elizabeth (2012): „The „Mediation“ of Muslim-Christian Relations in Egypt: the Strategies and Discourse of Official Egyptian Press during Mubarak's Presidency“. Islam and Christian-Muslim Relations 23.1: 31-44.

El-Khawaga, Dina (1992): „L'affirmation d'une identité chrétienne copte. Saisir un processus en cours“. In Itinéraires d’Égypte. Mélanges offerts au père Maurice Martin, s.j. Hrsg. Décobert, C. Kairo: Institut francais d’archéologie orientale du Caire, 345-365.

Al-Kiraza (2011a): „الكنيسة وسط التغييرات و الأحداث. (Die Kirche inmitten der Veränderungen und der Ereignisse)“ 1. April, Jahr 39. Nummer 1-2: 1. 
Al-Kiraza (2011b): بيان المجمع المقس الصادر من الكنيسة القبطية الأرثوذكسية. (Stellungnahme des Heiligen Synods herausgegeben von der koptisch-orthodoxen Kirche.“ 30. Dezember, Jahr 3. Nummer 5-6: 1.

Magdi, Mina (2015): 21. Jänner. Persönliches Interview. Kairo.

Mayeur-Jaouen, Catherine (2005): Pélerinages d'Égypte. Histoire de la piété copte et musulmane. XVe-XXe siècles. Paris: Éditions École des Hautes Études en sciences sociales.

McCallum, Fiona (2012): „Religious Institutions and Authoritarian States: Church-State Relations in the Middle East. Third World Quarterly 1.33: 109-124.

Murqus, Bischof (2015): 29. Jänner. Persönliches Interview. Kairo.

New York Times (2012): „Coptic Church chooses Pope Who rejects Political Role.“ 4. November. http://www.nytimes.com/2012/11/05/world/middleeast/coptic-church-chooses-popewho-rejects-politics.html?pagewanted = all\&_r=0. (17 Jänner 2017).

OASIS (2011): „On the Crest of the Tahrir Square, Interview with Antonios Naguib“. Jahr 7. Nummer 14: 24-27.

OASIS (2013): „Testimonies, Theology and Prayer: The 3 Pyramides of the Copts. Interview with Tawadros II“. Jahr 9. Nummer 17: 79-83.

Proche-Orient Chrétien (2011): Nummer 61.3-4.

Proche-Orient Chrétien (2012): Nummer 62.3-4.

Proche-Orient Chrétien (2013a): Nummer 63.1-2.

Proche-Orient Chrétien (2013b): Nummer 63.3-4.

Reiss, Wolfram (1998): Erneuerung in der koptisch-orthodoxen Kirche. Die Geschichte der koptisch-orthodoxen Sonntagsschulbewegung und die Aufnahme ihrer Reformansätze in den Erneuerungsbewegungen der koptisch-orthodoxen Kirche der Gegenwart. Hamburg: LIT.

Reiss, Wolfram (2013): „Die Situation der Kopten in der Gegenwart“. In: Kirche in Welt. Christentum in Zeiten kultureller Vielfalt. Hrsg. Hölschen, A. u.a. Frankfurt: Peter Lang Verlag, 45-90.

Roussillon, Alain (1994) : „Egypte, plus d’un demi-siècle d’activisme islamiste“. In: L'islamisme. Hrsg. Lahouari, A.; de Bièvres, C; Botiveau, B. Paris: La Découverte.

Roussillon, Alain (2010): „Les coptes à la marge“. Égypte/Monde arabe. http://ema.revues.org/ 3001. (17. Jänner 2017).

Al-Salah (2012a): صناعة تخويف المسيحيين. (Die Produktion der Angst)“ Nummer 9, 48.

Al-Salah (2012b): الأقباط ومخاوف الانتخابات بين التهوين والتهويل (Die Kopten und die Ängste vor den Wahlen. Zwischen Trivialisierung und Verängstigung)“, 31-37.

Sedra, Paul (2012a): „The Church, Maspero, and the Future of the Coptic Community“. Jadaliyya, 19. Mai. http://www.jadaliyya.com/pages/index/4735/the-church-maspero-and-thefuture-of-the-coptic-co. (17. Jänner 2017).

Sedra, Paul (2012b): „The Brotherhood's Politics of Fear and Division“. Egypt Independent, 23. Dezember. (http://www.egyptindependent.com/opinion/brotherhood-s-politics-fear-anddivision. (17. Jänner 2017).

Shenoda, M. M. (2007): „Displacing Dhimmī, Maintaining Hope: Unthinkable Coptic Representations of Fatimid Egypt“. International Journal of Middle East Studies, 39(4): 587-606.

Shenuda (2013 [1952]): مقال البابا شنودة بعد جر ائم الاخوان الطائفية بالسويس والزقازيق عام 1952 . (Artikel des Papstes Šinūda nach den konfessionellen Verbrechen der Muslimbruderschaft in Suez und al-Zaqāzīq im Jahre 1952)“. http://coptic-historian.blogspot.com/2013/09/1952.html (17. Jänner 2017).

Al-Shurouq (2011a). أقباط يتهمون قبادات سلفية ومحافظ أسوان بالتحريض على كنيسة المريناب (Die Kopten beschuldigen salafistische Anführer und den Gouverneur von Aswān der Anstiftung gegen die Kirche von al-Marīnāb)“ 5. Oktober, Nummer 977: 1. 
Al-Shurouq (2011b). مقتل 24 و إصابة العشرات في مواجهات بين الأقباط والأمن" (24 Tote und Dutzende Verletzte in Auseinandersetzung zwischen den Kopten und den Sicherheitskräften)“ 10. Oktober, Nummer 982: 1.

Al-Shurouq (2013): البابا يدخل خلوته ويمنع الاتصالات والمقابلات بالمسئولين ورجال الدين, Der Papst betritt seine Zurückgezogenheit und verbietet jeden Kontakt und Treffen mit den Verantwortlichen und dem Klerus.“ 12. April 2013, Nummer 1531: 4.

Sidhom, Youssef (2011): „Code Name: Sectarian Sedition“. Watani, 15. Mai, Jahr 11. Nummer 53: 3.

Tadros, Mariz (2013): Copts at the Crossroads: The Challenges of Building Inclusive Democracy in contemporary Egypt. Cairo; New York: The American University In Cairo Press.

Van Doorn-Harder, Nelly (2013): „The Copts in Egypt's Counter Revolution: On Being Egyptian“. Huffington Post, 15 November. (http://www.huffingtonpost.com/nelly-van-doornharder/ copts-in-egypt_b_3685400.html. (17. Jänner 2017).

Watani (2011a): 9. Jänner, Jahr 53, Nummer 2559, ganze Seite 5.

Watani (2011b): الكنائس تدعو الثعب للحفاظ على الوطن". (Die Kirchen rufen die Jugend dazu auf, das Vaterland zu erhalten)" 6. Februar, Jahr 53, Nummer 2563.3.

Watani (2011c): ,في ميدان التحرير : وفقة بالثموع لإحياء ذكرى أربعين شهاداء الإسكندرية. (Am Tahrir-Platz: Protest mit Kerzen zum Andenken an der 40 Märytrern von Alexandria)“ 13. Februar, Nummer 2564, Jahr 53, 3.

Watani (2011d): , الأقباط و المسلمون أقاموا صلوات واحدة من أجل مصر. (Kopten und Muslime beten zusammen für Äygpten)“ 13. Februar, Jahr 53, Nummer 2564: 4.

Watani (2011e): المبادرة الإخو انية للحوار مع شباب الأقباط.نعم ولكن. (Die Dialoginitiative der Muslimbruderschaft mit der koptischen Jugend ...ja, aber“ 17. April, Jahr 53, Nummer 2573: 5.

Watani (2011f): „Surrounded and attacked“. 15. Mai, Jahr 11, Nummer 543: 3.

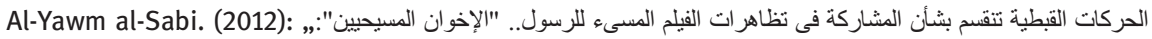

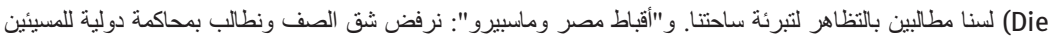
koptischen Bewegungen sind gespalten ob der Teilnahme an den Protesten gegen den Film, der den Propheten beleidigt. Die "Christlichen Brüder": wir fordern nicht zu Protesten, um uns von unserem Platz zu distanzieren. Die Kopten Ägyptens und Maspero: "Wie lehnen die Spaltung der Ränge ab und fordern einen internationalen Prozess gegen die Beleidigenden).“ 11 September. http://www.youm7.com/story/2012/9/11/\%D8\%A7\%D9\%84\%D8\%AD \%D8\%B1\%D9\%83\%D8\%A7\%D8\%AA_\%D8\%A7\%D9\%84\%D9\%82\%D8\%A8\%D8\%B7\%D9\% 8A\%D8\%A9_\%D8\%AA\%D9\%86\%D9\%82\%D8\%B3\%D9\%85_\%D8\%A8\%D8\%B4\%D8\%A3\% D9\%86_\%D8\%A7\%D9\%84\%D9\%85\%D8\%B4\%D8\%A7\%D8\%B1\%D9\%83\%D8\%A9_\%D9\% 81\%D9\%89_\%D8\%AA\%D8\%B8\%D8\%A7\%D9\%87\%D8\%B1\%D8\%A7\%D8\%AA_\%D8\%A7\% D9\%84\%D9\%81\%D9\%8A\%D9\%84\%D9\%85_\%D8\%A7\%D9\%84\%D9\%85\%D8\%B3\%D9\% 89\%D8\%A1/782863\#.Vjx7ZV50fVJ (1. Juli 2017).

Youssef, Fadi (2015): 20. Jänner. Persönliches Interview. Kairo.

YouTube (2012): رئيس حزب النور يتأسف لعدم تقدم النصارى للترشح على قو ائمهِ. (Der Präsident der Ḥizb unNūr bedauert, dass keine christlichen Kandidaten auf seiner Liste sind)“ Jänner. https:// www.youtube.com/watch?v=vTO2As_h-No. (17. Jänner 2017). 